\title{
Nuevos avances en la formación de fases interestratificadas durante el proceso de deshidratación-rehidratación de vermiculitas-Mg: Influencia de la presión de vacío, temperatura y composición
}

\author{
C. MARCOS ${ }^{1}$, A. RUÍZ-CONDE², A. ARGÜELLES',3, P. J. SÁNCHEZ-SOTO², A. GARCÍA', J.A. BLANCO \\ ${ }^{1}$ Dpto. Geología e Instituto de Organometálica Enrique Moles, Universidad de Oviedo. 33005, Oviedo, Spain. \\ ${ }^{2}$ Instituto de Ciencia de Materiales de Sevilla, Instituto Mixto Consejo Superior de Investigaciones Científicas (C.S.I.C.)-Universidad de Sevilla, \\ Isla de la Cartuja, 41092-Sevilla, Spain \\ ${ }^{3}$ Dpto. Física, Universidad de Oviedo, 33005, Oviedo, Spain.
}

\begin{abstract}
Se presentan nuevos resultados obtenidos mediante difracción de rayos $\mathrm{X}$ sobre las transformaciones estructurales que se producen en vermiculitas (en polvo y en lámina de exfoliación) con distinta composición y procedencia, cuando se las somete a condiciones diferentes de presión y temperatura. El proceso de deshidratación de las vermiculitas que se produce bajo vacío es similar al que tiene lugar con aumento de la temperatura, es decir, se lleva a cabo mediante la formación de fases interestratificadas, aunque su evolución es diferente ya que nunca se alcanza el estado de hidratación de cero capas de agua en el espacio interlaminar. La muestra de Sta. Olalla (Huelva), con el mayor contenido de magnesio en dicho espacio, muestra el comportamiento más complejo.
\end{abstract}

Palabras clave: Vermiculita, vacío, fases interestratificadas, difracción de rayos X.

New advances in the formation of interestratified phases during the dehydration-rehydration process of vermiculites: influence of vacuum pressure, temperature and composition.

New results obtained from X-ray diffraction about structural transformations induced by vacuum between the different hydration states of three vermiculites (powdered and flake samples) with different composition and origin, are presented. The dehydration process of the vermiculites under vacuum has been proved to be the same as that of temperature but with a different evolution through the different hydration states, although the zero-water layer hydration state has been not observed. The sample from Sta. Olalla, with the major content of $\mathrm{Mg}$ in the interlayer, exhibits the most complex behaviour.

Key words: Vermiculite, vacuum, interstratified phases, X-ray diffraction.

\section{INTRODUCCIÓN}

La vermiculita es un silicato laminar hidratado con aluminio y magnesio y cationes de cambio en el espacio interlaminar. Presenta interesantes propiedades y aplicaciones como materia prima, destacando la preparación de materiales cerámicos y vítreos, además de agregados ligeros y refractarios para la industria siderúrgica (1). El estado de hidratación de las vermiculitas queda definido por el número de capas de agua en el espacio interlaminar (estados de 2,1 y 0 capas de agua, respectivamente), con un espaciado interplanar que depende de la presión de vapor del agua, de la temperatura y de la composición. Para vermiculitas- Mg estos espaciados son $14.40 \AA ̊$ para el estado 2, $11.50 \AA ̊$ A para el estado 1 y 9.02 Å para el estado 0 (2).

En el presente trabajo se ha estudiado el proceso de deshidrataciónrehidratación de vermiculitas con diferente contenido de magnesio en el espacio interlaminar y diferente procedencia, en condiciones ambientales y con vacío $\left(1.4^{*} 10^{-2}\right.$ y $\left.2.4{ }^{*} 10^{-4} \mathrm{mbar}\right)$. Posteriormente se ha analizado la evolución de los diferentes estados de hidratación con el tiempo y la temperatura y se han comparado con los observados a presión ambiental anteriormente (3-5).

\section{MATERIALES Y MÉTODOS EXPERIMENTALES}

Las vermiculitas estudiadas proceden de Sta. Olalla (Huelva, España), Paulistana (Piauí, Brasil) y de la zona occidental de China, respectivamente.
Los análisis químicos se han realizado con una microsonda electrónica CAMEBAX-MBXSX-50 de los Servicios Científico-Técnicos de la Universidad de Oviedo. Los diagramas DRX se obtuvieron con un difractómetro Seifert XRD 3000 de los Servicios Científico-Técnicos de la Universidad de Oviedo. Las condiciones de uso fueron de $30 \mathrm{~mA} \mathrm{y}$ $40 \mathrm{kV}$ (radiación $\mathrm{Cu}-\mathrm{K} \alpha, \lambda=1.5418 \AA$ ), rango $2 \theta$ entre $2-70^{\circ}$, velocidad $0.02^{\circ} 2 \theta / 20 \mathrm{~s}$. Para los experimentos con vacío se utilizaron dos bombas comerciales Leybold (Trivac D $2.5 \mathrm{E}$ (hasta $10^{-2}$ mbar) y Turbovac TMP 50 (hasta $10^{-4}$ mbar). Los experimentos se han realizado tanto en muestra en polvo como en láminas de exfoliación.

\section{RESULTADOS Y DICUSIÓN}

\subsection{Análisis químicos}

A partir del porcentaje en peso de los óxidos de los elementos de las tres muestras se ha obtenido la fórmula estructural de las mismas (Tabla I) con el programa MINPET (MINPET GEOLOGICAL SOFTWARE, 146 DV Chateau Masson-Angers, Quebec, Canada), en base a 24 aniones. La muestra de Sta. Olalla es muy pura, compuesta exclusivamente por vermiculita, como se deduce del contenido en $\mathrm{K}_{2} \mathrm{O}$ (6) y del de $\mathrm{Mg}^{2+}$ del espacio interlaminar de su estructura. El contenido en $\mathrm{K}_{2} \mathrm{O}$ de la muestra de Piaui es mayor y el del $\mathrm{Mg}^{2+}$ del espacio interlaminar menor. Por último, la muestra de China tiene una composición muy diferente a la de una vermiculita típica. 
TABla I. Fórmula estructural de las Vermiculitas de Sta. Olalla, PiAUI Y CHINA.

\begin{tabular}{|l|l|}
\hline Muestras & Fórmula estructural \\
\hline Sta. Olalla & $\left(\mathrm{Mg}_{0.75} \mathrm{Ca}_{0.05} \mathrm{Na}_{0.04}\right)\left(\mathrm{Mg}_{4.92} \mathrm{Al}_{0.59} \mathrm{Fe}_{0.43} \mathrm{Ti}_{0.04}\right)\left(\mathrm{Si}_{5.66} \mathrm{Al}_{2.34}\right) \mathrm{O}_{20}(\mathrm{OH})_{4}$ \\
\hline Piaui & $\left(\mathrm{Mg}_{0.55} \mathrm{~K}_{0.69} \mathrm{Ca}_{0.03} \mathrm{Na}_{0.01}\right)\left(\mathrm{Ti}_{0.13} \mathrm{Fe}_{0.61} \mathrm{Cr}_{0.01} \mathrm{Mn}_{0.01} \mathrm{Mg}_{5.24}\right)\left(\mathrm{Si}_{6.10} \mathrm{Al}_{1.67} \mathrm{Fe}_{0.24}\right) \mathrm{O}_{20} \mathrm{OH}_{4}$ \\
\hline China & $\left(\mathrm{K}_{1.17} \mathrm{Ca}_{0.05} \mathrm{Na}_{0.17}\right)\left(\mathrm{Ti}_{0.09} \mathrm{Mg}_{4.43}\right)\left(\mathrm{Si}_{4.88} \mathrm{Al}_{1.71}\right) \mathrm{O}_{20} \mathrm{OH}_{4}$ \\
\hline
\end{tabular}

\subsection{Estudio de difracción de rayos $X$}

Los difractogramas obtenidos para las tres muestras en condiciones ambientales se muestran en la Figura 1. Se observa que las muestras de Sta. Olalla y Piaui presentan las reflexiones típicas de las vermiculitas, con la reflexión más intensa 002 a un valor de $\mathrm{d}=14.2 \AA$ para la primera y $14.4 \AA$ Å para la segunda. Por el contrario, la reflexión más intensa en la muestra de China está asociada con la estructura tipo flogopita y las reflexiones correspondientes a la vermiculita son menos intensas, debido a su menor cristalinidad.

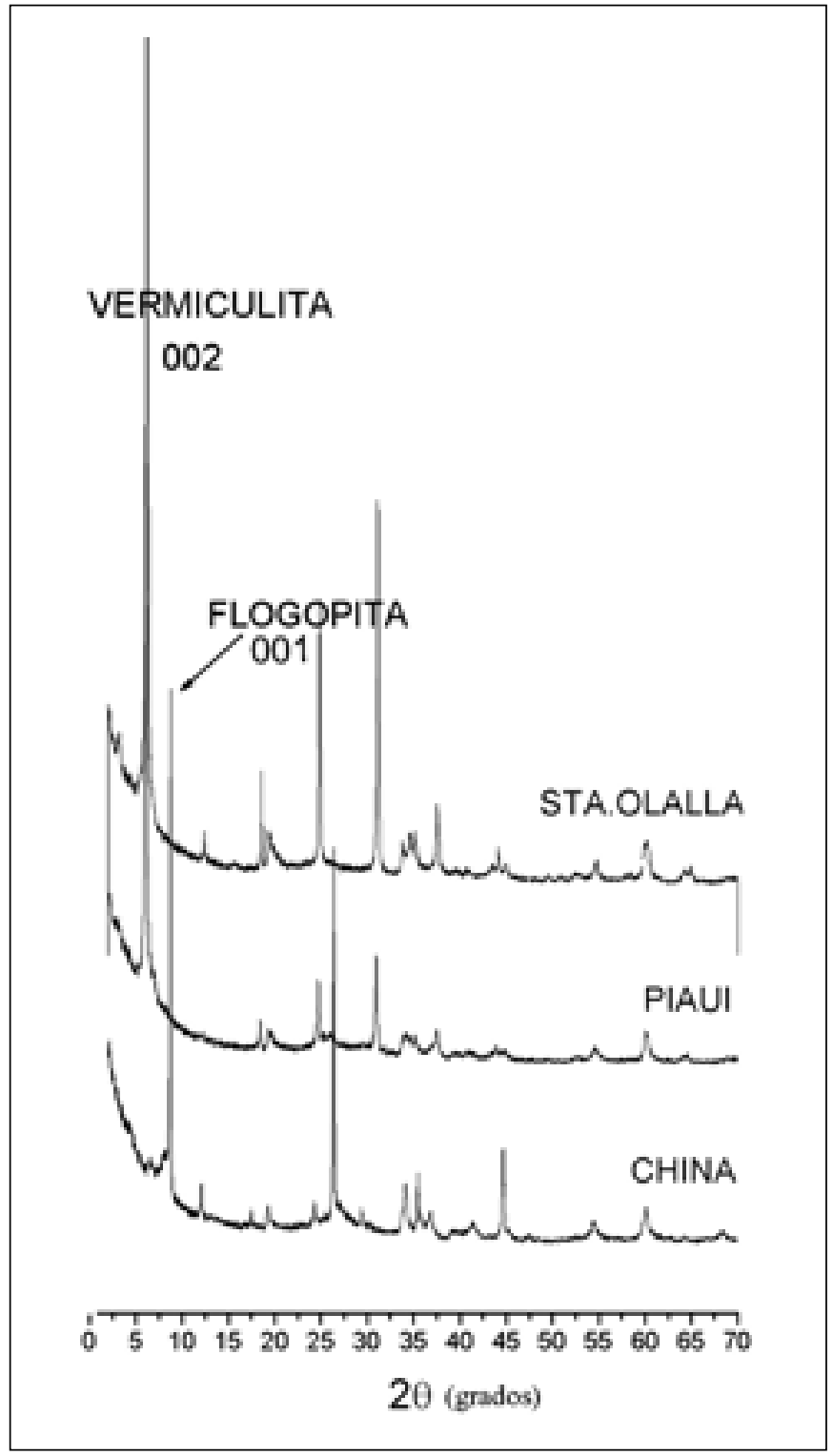

Figura 1.- Difractogramas de las vermiculitas de Sta. Olalla, Piauí y China a temperatura y presión ambiental.
Los difractogramas para la muestra de Sta. Olalla en polvo a $\mathrm{P}$ ambiental y temperaturas entre 30 y $120^{\circ} \mathrm{C}$ se muestran en la Figura 2. Los estados de hidratación presentes concuerdan con los observados por (4) y (5). La coexistencia entre ellos, aunque con distancias interplanares diferentes a las obtenidas por nosotros, ha sido previamente observada por (3).

Los difractogramas obtenidos para las muestras en polvo, a diferentes valores de presión de vacío y temperaturas muestran que la primera es la única que afecta a la deshidratación de las vermiculitas, modificando su estructura cristalina a través de la formación de fases interestratificadas (Figura 3). El efecto es el mismo que se produce con la variación de temperatura en el rango de 120 a $140^{\circ}(5)$.

El proceso de deshidratación cuando se aplica el vacío, a temperatura ambiente, depende de la presión aplicada, la composición y la secuencia de ambientes en la realización del experimento. En la Figura 3 se puede apreciar la diferencia en los difractogramas obtenidos con presiones diferentes (ambiente y $1.4^{*} 10^{-2}$ mbares) a $27^{\circ} \mathrm{C}$ para la muestra en polvo de Sta. Olalla. El efecto del vacío con la composición se pone de manifiesto en los difractogramas obtenidos a presión ambiental (Figura

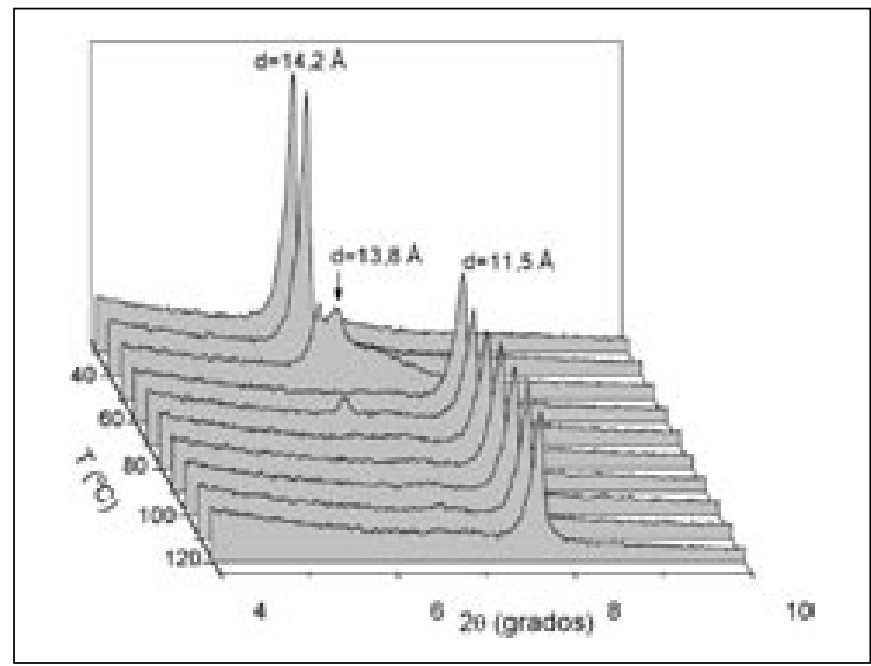

Figura 2.- Difractogramas de la vermiculita en polvo de Sta. Olalla a presión ambiente y alta temperatura $\left(30^{\circ} \mathrm{C}-120^{\circ} \mathrm{C}\right)$ mostrando la coexistencia de estados hidratados con distancias interplanares diferentes.

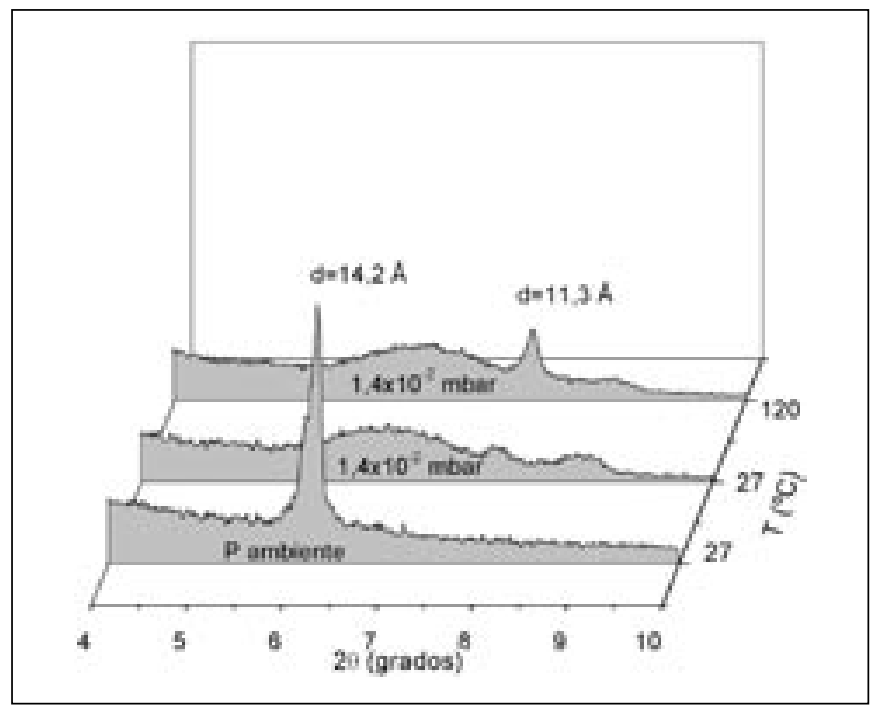

Figura 3.- Difractogramas de la muestra en polvo de Sta. Olalla mostrando la formación de fases interestratificadas y su variación con la presión de vacío y la temperatura. 
4a) y a $1.4^{\star} 10^{-2}$ mbar (Figura $4 b$ ) de vermiculitas en lámina de exfoliación de Sta. Olalla, Piaui y China, con diferente composición química como se expuso en el apartado anterior. Queda patente la evolución de la reflexión 002 y la presencia de fases interestratificadas. Estas fases interestratificadas han sido confirmadas mediante la aplicación del método directo de la transformada de Fourier (7), de forma análoga a como se realizó en un trabajo previo (5). En la Figura 5 se puede observar la evolución de la estructura cristalina en función de la secuencia seguida en la aplicación del vacío, es decir, según que la muestra haya sido medida bajo vacío después de haberlo hecho a presión ambiente o no. A medida que aumenta la presión de vacío la reflexión 002 de la vermiculita se desplaza hacia valores más altos de $2 \theta$ y su intensidad disminuye, significa que la vermiculita pasa del estado de hidratación con 2 capas de agua en el espacio interlaminar a $\mathrm{P}$ ambiente al estado con 1 capa de agua cuando la $\mathrm{P}$ de vacío disminuye. El proceso es más espontáneo en las muestras en polvo que en las de lámina de exfoliación.

\section{CONCLUSIONES}

Cuando las vermiculitas se someten a calentamiento en condiciones de vacío, el proceso de deshidratación es muy diferente del que sufren cuando son sometidas a tratamiento térmico hasta $120^{\circ} \mathrm{C}$. Las muestras se deshidratan justo cuando se aplica el vacío, sin tener efecto la temperatura.

El proceso de deshidratación producido bajo vacío depende de factores, como el valor de la presión de vacío, composición y secuencia de ambientes en la realización del experimento. El estado de hidratación con 1 capa de agua en el espacio interlaminar se consigue más fácilmente en la vermiculita de Piauí que en la de Sta. Olalla, debido a un menor contenido de $\mathrm{Mg}^{2+}$ y la afinidad por las moléculas de agua de este catión. La vermiculita de China no muestra la misma evolución del estado de hidratación que las otras dos muestras, debido a su diferente estructura cristalina puesta de manifiesto en el diagrama de rayos $X$.

\section{AGRADECIMIENTOS}

Se agradece la financiación recibida al Proyecto FICYT: FC-02-PCCIS01-42 que ha permitido la realización del trabajo, así como al III Plan Andaluz de Investigación de la Junta de Andalucía (Grupo TEP 204).

\section{BIBLIOGRAFÍA}

1. M.J. Potter, «Vermiculite: Annual Minerals Review», Am. Ceram. Soc. Bull. 81, 159-161 (2002).

2. Walker, G.F. Mechanism of dehydration of Mg-vermiculite. Clays and Clay Minerals, 4, 101 (1956).

3. Collins, D.R., Fitch, A.N. \& Catlow, R.A. «Dehydration of vermiculites and montmorillonites: a time-resolved powder neutron difraction study». Journal of Material Chemistry, 8, 865-873 (1992)

4. Reichenbach, H.G. \& Beyer, J. «Dehydration and rehydration of vermiculites: IV. Arrangements of interlayer components in the $1.43 \mathrm{~nm}$ and $1.38 \mathrm{~nm}$ hydrates of Mg-vermiculite». Clay Minerals, 29, 327-340 (1994).

5. Ruiz-Conde, A.; Ruiz-Amil, A.; Pérez-Rodríguez, J.L. Sánchez-Soto, P.J. «Dehydration-rehydration in magnesium vermiculite: conversion from two-one and one-two water hydration states through the formation of interstratified phases». Journal of Material Chemistry, 6, 1557-1566 (1996).

6. Velde, B. «High temperature or metamorphic vermiculites». Contributions to Mineralogy and Petrology, 66, 319-323 (1978).

7. Marcos, C., Argüelles, A., Ruíz-Conde, A., Sánchez-Soto, P.J., Blanco, J.A. «Study of the dehydration process of vermiculites by applying a vacuum pressure: formation of interstratified phases». Mineralogical Magazine, Vol. 67(6), 1253-1268 (2003).

Recibido: 1.2 .03

Aceptado: 30.11 .03

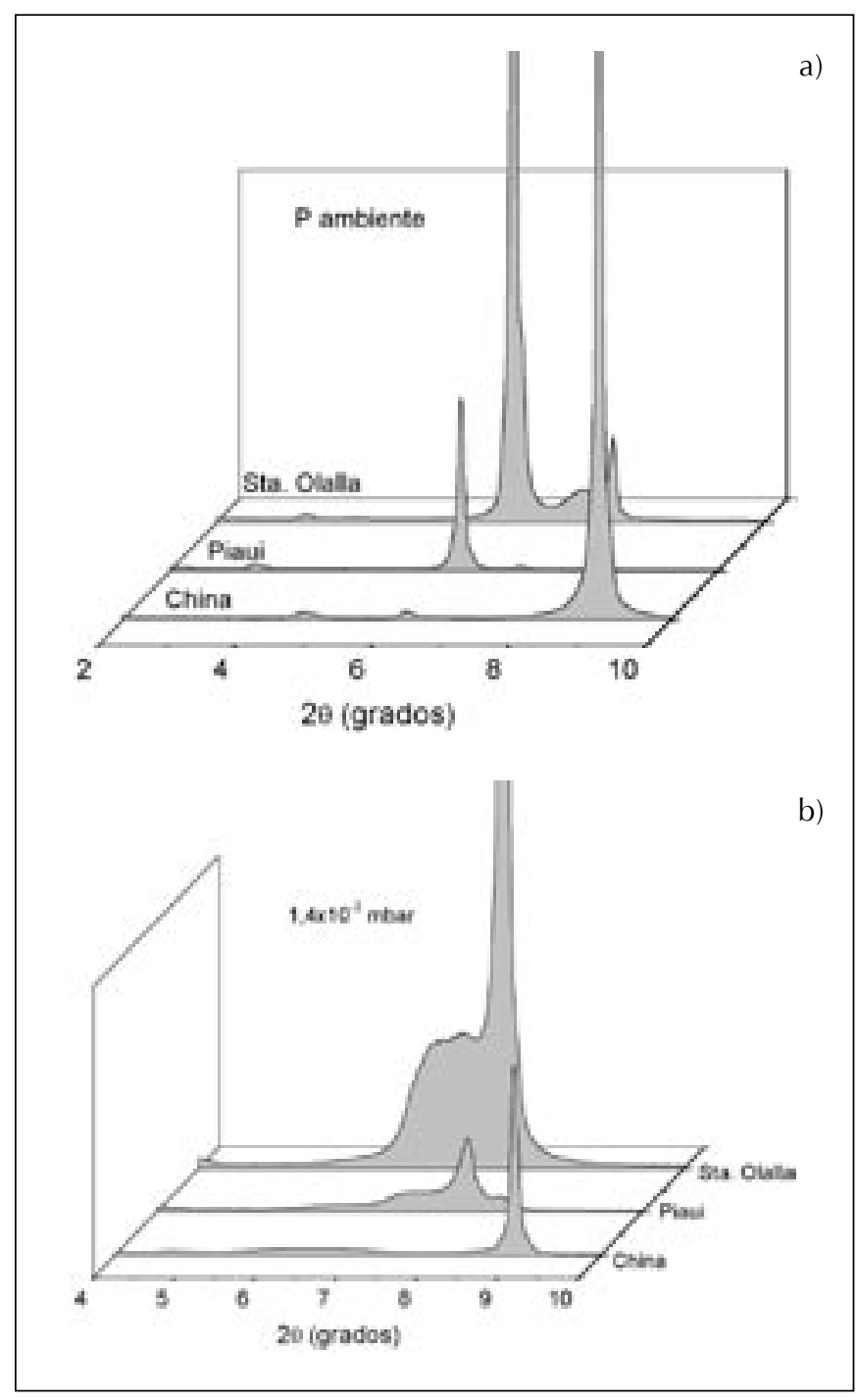

Figura 4.- Difractogramas de vermiculitas en lámina de exfoliación de Sta. Olalla, Piaui y China (a, a presión ambiente; b, con un vacío de $1,4 \times 10^{-2}$ mbar) mostrando el efecto del vacío con la composición, a temperatura ambiente.

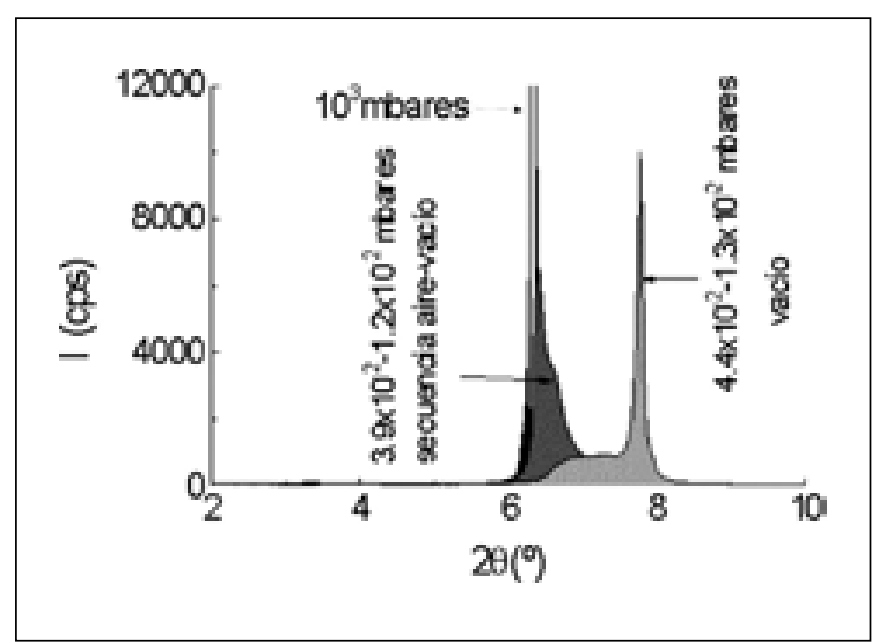

Figura 5.- Difractogramas mostrando la evolución de la estructura cristalina de la vermiculita de Sta. Olalla en función de la secuencia seguida en la aplicación del vacío. 